\title{
Recognition of Text CAPTCHA by using Back Propagation Algorithm of Artificial Neural network
}

\author{
Sumeet Gill, Renu Saroha
}

\begin{abstract}
Text-based CAPTCHA is a very simple type of CAPTCHA which are most widely used. It uses only a group of characters. In this paper, we focus on how Text based CAPTCHA is recognized by machine learning techniques. This paper proposed a method based on Back Propagation algorithm to identify the Text based CAPTCHA. The proposed technique improves the security level of Text-based CAPTCHA storage system by using the Back-propagation method of Artificial Neural Network. We used NNToolbox to train the network in MATLAB software.
\end{abstract}

Keywords: Text-based CAPTCHA, Back-propagation Algorithm, Artificial Neural Networks.

\section{INTRODUCTION:}

Now a days, all work is mostly done on the internet like education, shopping, net banking, and social discussion. On the majority of these sites, users need to fill their registration form by entering all the personal information in order to surf that particulars sites and different kinds of other survey forms. Due to increased technology, hacking software are available to fill all details automatically. One of the most important fields in our modern technology is related to protecting the information and securing it. The term CAPTCHA was invented by Luis Von Ahn, Manuel Blum, Nicholas J, Hopper and John Langford at Carnegie Mellon University (CMU) in 2000 [1]. CAPTCHA is an important standard internet security technique to protect email and other services from bots attack. It is successfully applied a number of application domains including education, business, social sites, and etc. CAPTCHA is "Completely automated public Turing test". Turing test is the standard interpretation of the behaviour of a machine in certain fixed options where the machine is to make decisions based on the inputs presented to it [2]. A good example of standard interpretation is as follows:

Revised Manuscript Received on July 22, 2019

Sumeet Gill, Associate Professor in Department of Mathematics, Maharshi Dayan and University, Rohtak-124001, Haryana (India)

Renu Saroha, Ph.d Scholar Department of Computer Science Application, Maharshi Dayan and University, Rohtak-124001, Haryana (India)
Suppose we have three players A, B, and C. Player C is the interrogator, who has given the test of guessing which player (player A or Player B) is a computer and which is human. Automated means to quickly perform the measurement and evaluate the test result. Turing test is for the standard interpretation of the behaviour of a machine in a certain fixed option where the machine is to make the decision based on the inputs presented to it. It is a security test that differentiates a computer and human apart. CAPTCHA code is a new network security technology. This technology is related to the protection of information and provides a way of securing it. It is also known as human interactive proof system that is used to secure our application on the internet. The normal scenario for providing protection and security against attacks is to use unique username and password, but this kind of security is normally breached and various attacks are being performed by different programs and various software are used for applying automatic attacks. CAPTCHA prevents the entry of automated script known as Bots. Bots are usually a robot which replicates the behaviour of humans and carries out malicious activities on web applications. Internet bots are also known as a web robot. It is a software application that runs automated tasks over the internet. The main advantage of CAPTCHA is that it is difficult for computers to extract the texts form such as distorted or puzzled images whereas it is easy for humans to understand the text hidden behind the distortion. Text-Based CAPTCHAs are randomly generated and are very simple to implement. It is a very effective technique and requires a large question bank. Text CAPTCHAs are displayed to the user during the signing up process. Text-based CAPTCHA includes simple type of question like, What is $5+4=$ ?, What is $6 * 7=$ ?, Which one among apple, curd, table, pen is fruit?, Enter the number thirteen, hundred forty-three?, How many colors are there in a rainbow?, Which is $2^{\text {nd }}$ in the series 35,19 and thirtyfour?, Which one among socks, library, cake or red is a colours? Text-based CAPTCHA is seen at the bottom of any application and registration form. CAPTCHAs faces a lot of problems like brute force attacks, relay attacks, dictionary attacks, denial-of-service attacks and, etc. Textbased CAPTCHAs are easily recognized by OCR (Optical Character Recognition). These are easy for a tester machine to generate and grade but hard for a software robot to pass.

As studied in previous year papers, to recognize the text Convolution Neural Network algorithm is used [8]. This algorithm includes three steps to recognize, these are: preprocessing, segmentation and recognition of images. In this paper, the Back propagation algorithm is used and it does not require pre-processing, segmentation and recognition of images. It 


\begin{tabular}{|c|c|c|c|c|c|}
\hline zitge & jqqan & Zilgh & & $\underline{D z y H n}$ & Fyval \\
\hline Ggt3f & & & Halt2 & & \\
\hline Of6th & Pulot & 3Wnliq & & Mqu45 & Taqu4 \\
\hline Othis & roscoe & Jb4n2 & & emper & Senec \\
\hline
\end{tabular}

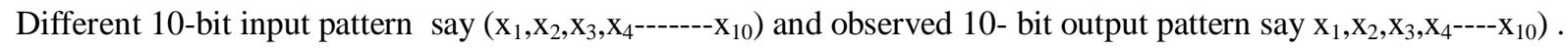

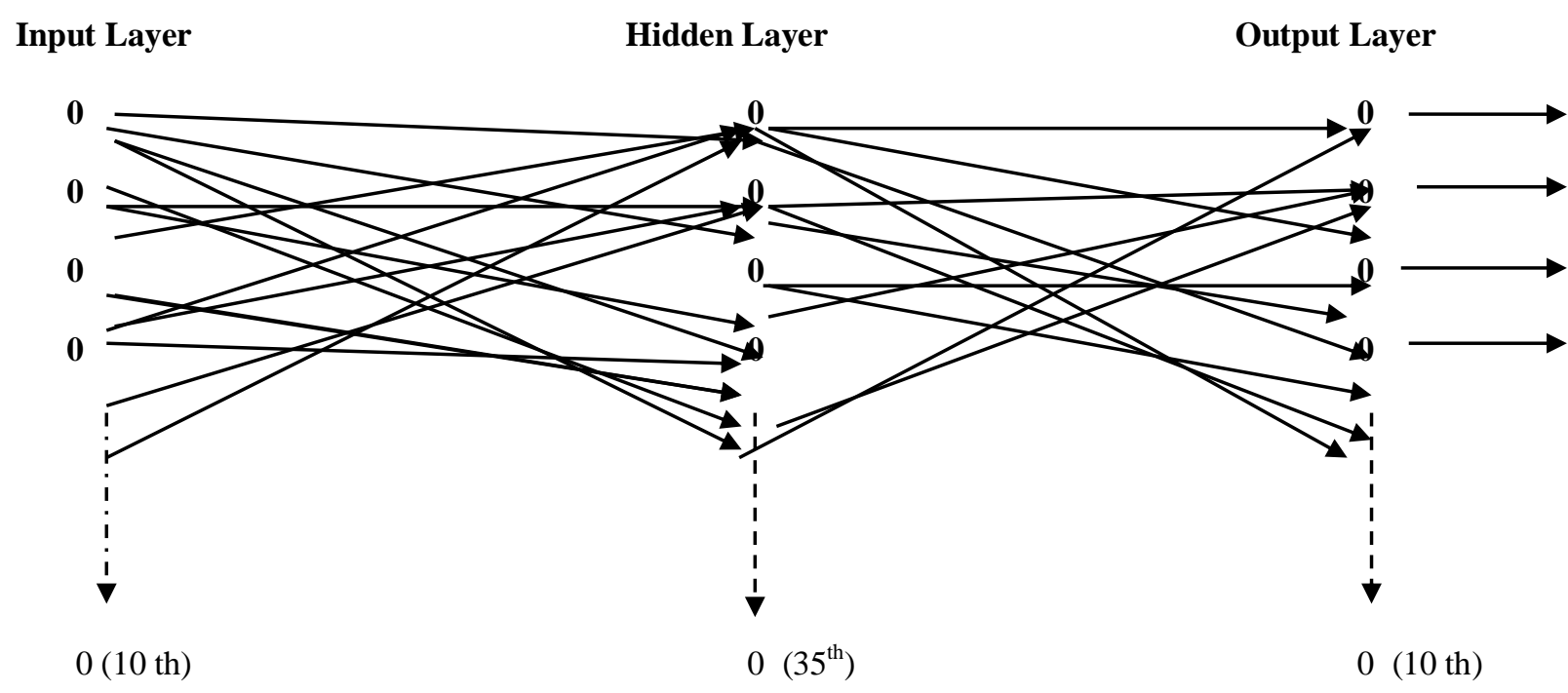

Figure1 Shows Neural Network Architecture for a Feed Forward Network

The input and output values of the network are represented as $S_{i}$ and $S_{0}$. The activation output information for the input layer is $\mathrm{x}_{\mathrm{i}}$ and for the output layer is $y_{j}$. The output activation for the hidden layer is $\mathrm{S}_{\mathrm{h}}$ and $\mathrm{q}_{\mathrm{h}}$. The output signal and the activation values for the input, hidden and output layer are represent as for the $1^{\text {th }}$ pattern in $\mathrm{m}^{\text {th }}$ iteration.

\section{Experimental Design}

In this experiment, we are performing the actual training and experiment conducted on dummy data. Here we memorize the input pattern sets by minimizing the error between the as training data set desired and actual output for the 10 input set and 10 output set. The last 40 bits are being used as test patterns. In this experiment, we estimate the password storage with standard Back Propagation Model. The experiment described is designed to achieve the encryption pattern storage in the network. The experiment has been conducted in MATLAB 2016.

The initial parameters taken before training for formation on network are given in table below:

\begin{tabular}{|l|l|}
\hline Parameters & Value \\
\hline Neurons in input layer & 10 \\
\hline Number of hidden layers & 1 \\
\hline Neurons in hidden layer & 1 \\
\hline
\end{tabular}




\begin{tabular}{|l|l|}
\hline Neurons in output layer & 10 \\
\hline Minimum error exist in the Network & 0.001 \\
\hline Initial Weights and biased term values & Values between 0 and 1 \\
\hline
\end{tabular}

\section{Table1 : Parameters used for Training of Network using Back Propagation Model}

\section{Input pattern sets}

Training of the system was carried out for following 10 input pattern sets:

\begin{tabular}{|c|c|c|c|c|c|c|c|c|c|}
\hline 1 & 1 & 1 & 1 & 1 & 1 & 1 & 0 & 1 & 1 \\
\hline 0 & 0 & 0 & 0 & 0 & 0 & 1 & 1 & 1 & 1 \\
\hline 1 & 0 & 1 & 0 & 0 & 0 & 0 & 0 & 0 & 0 \\
\hline 1 & 1 & 1 & 0 & 0 & 0 & 1 & 0 & 1 & 1 \\
\hline 0 & 0 & 0 & 1 & 1 & 1 & 0 & 0 & 1 & 0 \\
\hline 1 & 1 & 1 & 0 & 1 & 1 & 1 & 0 & 1 & 0 \\
\hline 0 & 0 & 0 & 0 & 0 & 1 & 1 & 1 & 1 & 1 \\
\hline 1 & 1 & 1 & 1 & 1 & 1 & 1 & 1 & 1 & 1 \\
\hline 0 & 0 & 0 & 0 & 0 & 0 & 1 & 0 & 1 & 1 \\
\hline 0 & 1 & 0 & 1 & 1 & 1 & 0 & 0 & 1 & 0 \\
\hline 1 & 0 & 1 & 1 & 0 & 0 & 0 & 1 & 1 & 1 \\
\hline 0 & 0 & 0 & 0 & 1 & 1 & 0 & 0 & 0 & 1 \\
\hline 1 & 0 & 1 & 1 & 1 & 1 & 1 & 0 & 0 & 1 \\
\hline 0 & 1 & 0 & 0 & 0 & 0 & 0 & 1 & 0 & 1 \\
\hline 1 & 1 & 1 & 1 & 1 & 1 & 1 & 1 & 0 & 1 \\
\hline 0 & 0 & 0 & 0 & 0 & 0 & 1 & 0 & 1 & 1 \\
\hline 1 & 1 & 0 & 1 & 0 & 0 & 1 & 1 & 1 & 1 \\
\hline 0 & 0 & 1 & 1 & 0 & 0 & 1 & 0 & 0 & 0 \\
\hline 1 & 0 & 1 & 0 & 0 & 1 & 0 & 1 & 0 & 0 \\
\hline 0 & 0 & 0 & 0 & 0 & 0 & 0 & 0 & 1 & 0 \\
\hline 0 & 1 & 0 & 1 & 1 & 0 & 0 & 0 & 1 & 0 \\
\hline 1 & 1 & 1 & 1 & 1 & 1 & 1 & 1 & 0 & 1 \\
\hline 0 & 0 & 0 & 0 & 0 & 0 & 1 & 0 & 1 & 1 \\
\hline 0 & 0 & 0 & 0 & 1 & 1 & 0 & 1 & 0 & 0 \\
\hline 0 & 0 & 0 & 1 & 1 & 1 & 1 & 0 & 1 & 1 \\
\hline 1 & 0 & 1 & 0 & 0 & 0 & 1 & 1 & 0 & 0 \\
\hline 1 & 1 & 1 & 0 & 0 & 1 & 0 & 0 & 0 & 0 \\
\hline 1 & 1 & 1 & 0 & 1 & 0 & 1 & 1 & 1 & 1 \\
\hline 1 & 1 & 1 & 1 & 1 & 1 & 1 & 1 & 1 & 1 \\
\hline 0 & 0 & 0 & 0 & 0 & 0 & 1 & 0 & 1 & 1 \\
\hline 0 & 0 & 1 & 0 & 0 & 1 & 0 & 0 & 0 & 0 \\
\hline 0 & 1 & 1 & 1 & 1 & 1 & 1 & 0 & 1 & 0 \\
\hline 1 & 1 & 0 & 1 & 1 & 0 & 0 & 1 & 0 & 1 \\
\hline 1 & 1 & 1 & 1 & 0 & 1 & 0 & 1 & 1 & 0 \\
\hline 1 & 0 & 0 & 0 & 0 & 0 & 1 & 0 & 0 & 1 \\
\hline
\end{tabular}

Table2. Shows Input pattern dataset sets

\section{Test Pattern Sets}

The following pattern sets were chosen to be test patterns for recalling:

\begin{tabular}{|l|l|l|l|l|l|l|l|l|l|}
\hline 1 & 0 & 0 & 0 & 0 & 0 & 0 & 1 & 0 & 1 \\
\hline 0 & 0 & 1 & 0 & 0 & 1 & 1 & 0 & 1 & 0 \\
\hline 0 & 0 & 0 & 0 & 0 & 0 & 1 & 0 & 0 & 1 \\
\hline 0 & 1 & 1 & 1 & 1 & 1 & 0 & 1 & 1 & 1 \\
\hline
\end{tabular}

Determining of Coupling Strength between the Neuron Layers. 


\section{Recognition of Text CAPTCHA by using Back Propagation Algorithm of Artificial Neural network}

For estimating the training of encryption pattern of passwords, we first need to define the functional dependency between the encrypted passwords in the form of weights in the neural network.

\begin{tabular}{|c|c|c|c|c|c|}
\hline \multicolumn{3}{|c|}{ Between Input Layer to Hidden Layer } & \multicolumn{3}{|c|}{ Initial Weights } \\
\hline 0.39483 & -0.34012 & -0.61717 & 0.40563 & 0.48655 & -0.27982 \\
\hline-0.22963 & $\begin{array}{l}-0.46503 \\
\end{array}$ & 0.28964 & $-0.30761 ;$ & -0.22963 & -0.46503 \\
\hline 0.36838 & 0.59305 & 0.14725 & 0.45316 & -0.16646 & -0.35268 \\
\hline 0.51488 & -0.19476 & -0.17803 & 0.01263 & -0.17999 & -0.21126 \\
\hline 0.39483 & -0.34012 & -0.61717 & 0.40563 & 0.48655 & -0.27982 \\
\hline-0.30761 & -0.22963 & 0.28964 & -0.30761 & -0.46503 & 0.28964 \\
\hline 0.36838 & 0.59305 & 0.14725 & 0.45316 & -0.16646 & -0.35268 \\
\hline I & & & I & $\begin{array}{r} \\
1 \\
1\end{array}$ & I \\
\hline $0.33097^{\nabla}$ & -0.43069 & -0.41724 & $-0.0488 \overline{4} 5$ & $-0.264 \overline{55}$ & $-0.459 \overline{47}$ \\
\hline 0.53351 & 0.13582 & -0.43691 & -0.065149 & 0.22913 & -0.042134 \\
\hline-0.090708 & 0.317 & 0.28739 & -0.52859 & -0.43479 & 0.2426 \\
\hline 0.42716 & 0.44417 & 0.081215 & -0.42247 & -0.11168 & -0.1049 \\
\hline-0.40321 & -0.28442 & -0.029918 & -0.61638 & -0.12866 & -0.28887 \\
\hline 0.33097 & -0.43069 & -0.41724 & -0.048845 & -0.26455 & -0.45947 \\
\hline
\end{tabular}

Table4. Shows the weights matrix of Test pattern sets.

\section{Results}

\section{Memorised Pattern Set}

The network was trained for following 10 input output pattern sets.

\begin{tabular}{|c|c|c|c|c|c|c|c|c|c|c|c|c|c|c|c|c|c|c|c|c|}
\hline 1 & 1 & 1 & 1 & 1 & 1 & 1 & 1 & 0 & 1 & 1 & 1 & 1 & 1 & 1 & 1 & 1 & 1 & 0 & 1 & 1 \\
\hline 2 & 0 & 0 & 0 & 0 & 0 & 0 & 1 & 1 & 1 & 1 & 0 & 0 & 0 & 0 & 0 & 0 & 1 & 1 & 1 & 1 \\
\hline 3 & 1 & 0 & 1 & 0 & 0 & 0 & 0 & 0 & 0 & 0 & 1 & 0 & 1 & 0 & 0 & 0 & 0 & 0 & 0 & 0 \\
\hline 4 & 1 & 1 & 1 & 0 & 0 & 0 & 1 & 0 & 1 & 1 & 1 & 1 & 1 & 0 & 0 & 0 & 1 & 0 & 1 & 1 \\
\hline 5 & 0 & 0 & 0 & 1 & 1 & 1 & 0 & 0 & 1 & 0 & 0 & 0 & 0 & 1 & 1 & 1 & 0 & 0 & 1 & 0 \\
\hline 6 & 1 & 1 & 1 & 0 & 1 & 1 & 1 & 0 & 1 & 0 & 1 & 1 & 1 & 0 & 1 & 1 & 1 & 0 & 1 & 0 \\
\hline 7 & 0 & 0 & 0 & 0 & 0 & 1 & 1 & 1 & 1 & 1 & 0 & 0 & 0 & 0 & 0 & 1 & 1 & 1 & 1 & 1 \\
\hline 8 & 1 & 1 & 1 & 1 & 1 & 1 & 1 & 1 & 1 & 1 & 1 & 1 & 1 & 1 & 1 & 1 & & 1 & 1 & 1 \\
\hline 9 & 0 & 0 & 0 & 0 & 0 & 0 & 1 & 0 & 1 & 1 & 0 & 0 & 0 & 0 & 0 & & & & 1 & 1 \\
\hline
\end{tabular}




\begin{tabular}{|l|l|l|l|l|l|l|l|l|l|l|l|l|l|l|l|l|l|l|l|l|l|}
\hline 10 & 0 & 1 & 0 & 1 & 1 & 1 & 0 & 0 & 1 & & 0 & 0 & 1 & 0 & 1 & 1 & 1 & 0 & 0 & 1 & 0 \\
\hline 11 & 1 & 0 & 1 & 1 & 0 & 0 & 0 & 1 & 1 & & 1 & 1 & 0 & 1 & 1 & 0 & 0 & 0 & 1 & 1 & 1 \\
\hline 12 & 0 & 0 & 0 & 0 & 1 & 1 & 0 & 0 & 0 & & 1 & 0 & 0 & 0 & 0 & 1 & 1 & 0 & 0 & 0 & 1 \\
\hline 13 & 1 & 0 & 1 & 1 & 1 & 1 & 1 & 0 & 0 & & 1 & 1 & 0 & 1 & 1 & 1 & 1 & 1 & 0 & 0 & 1 \\
\hline 14 & 0 & 1 & 0 & 0 & 0 & 0 & 0 & 1 & 0 & & 1 & 0 & 1 & 0 & 0 & 0 & 0 & 0 & 1 & 0 & 1 \\
\hline 15 & 1 & 1 & 1 & 1 & 1 & 1 & 1 & 1 & 0 & & 1 & 1 & 1 & 1 & 1 & 1 & 1 & 1 & 1 & 0 & 1 \\
\hline 16 & 0 & 0 & 0 & 0 & 0 & 0 & 1 & 0 & 1 & & 1 & 0 & 0 & 0 & 0 & 0 & 0 & 1 & 0 & 1 & 1 \\
\hline 17 & 1 & 1 & 0 & 1 & 0 & 0 & 1 & 1 & 1 & & 1 & 1 & 1 & 0 & 1 & 0 & 0 & 1 & 1 & 1 & 1 \\
\hline 18 & 0 & 0 & 1 & 1 & 0 & 0 & 1 & 0 & 0 & & 0 & 0 & 0 & 1 & 1 & 0 & 0 & 1 & 0 & 0 & 0 \\
\hline 19 & 1 & 0 & 1 & 0 & 0 & 1 & 0 & 1 & 0 & & 0 & 1 & 0 & 1 & 0 & 0 & 1 & 0 & 1 & 0 & 0 \\
\hline 20 & 0 & 0 & 0 & 0 & 0 & 0 & 0 & 0 & 1 & & 0 & 0 & 0 & 0 & 0 & 0 & 0 & 0 & 0 & 1 & 0 \\
\hline 21 & 0 & 1 & 0 & 1 & 1 & 0 & 0 & 0 & 1 & & 0 & 0 & 1 & 0 & 1 & 1 & 0 & 0 & 0 & 1 & 0 \\
\hline 22 & 1 & 1 & 1 & 1 & 1 & 1 & 1 & 1 & 0 & & 1 & 1 & 1 & 1 & 1 & 1 & 1 & 1 & 1 & 0 & 1 \\
\hline 23 & 0 & 0 & 0 & 0 & 0 & 0 & 1 & 0 & 1 & & 1 & 0 & 0 & 0 & 0 & 0 & 0 & 1 & 0 & 1 & 1 \\
\hline 24 & 0 & 0 & 0 & 0 & 1 & 1 & 0 & 1 & 0 & & 0 & 0 & 0 & 0 & 0 & 1 & 1 & 0 & 1 & 0 & 0 \\
\hline 25 & 0 & 0 & 0 & 1 & 1 & 1 & 1 & 0 & 1 & & 1 & 0 & 0 & 0 & 1 & 1 & 1 & 1 & 0 & 1 & 1 \\
\hline 26 & 1 & 0 & 1 & 0 & 0 & 0 & 1 & 1 & 0 & & 0 & 1 & 0 & 1 & 0 & 0 & 0 & 1 & 1 & 0 & 0 \\
\hline 27 & 1 & 1 & 1 & 0 & 0 & 1 & 0 & 0 & 0 & & 0 & 1 & 1 & 1 & 0 & 0 & 1 & 0 & 0 & 0 & 0 \\
\hline 28 & 1 & 1 & 1 & 0 & 1 & 0 & 1 & 1 & 1 & & 1 & 1 & 1 & 1 & 0 & 1 & 0 & 1 & 1 & 1 & 1 \\
\hline 29 & 1 & 1 & 1 & 1 & 1 & 1 & 1 & 1 & 1 & & 1 & 1 & 1 & 1 & 1 & 1 & 1 & 1 & 1 & 1 & 1 \\
\hline 30 & 0 & 0 & 0 & 0 & 0 & 0 & 1 & 0 & 1 & & 1 & 0 & 0 & 0 & 0 & 0 & 0 & 1 & 0 & 1 & 1 \\
\hline 31 & 0 & 0 & 1 & 0 & 0 & 1 & 0 & 0 & 0 & & 0 & 0 & 0 & 1 & 0 & 0 & 1 & 0 & 0 & 0 & 0 \\
\hline 32 & 0 & 1 & 1 & 1 & 1 & 1 & 1 & 0 & 1 & & 0 & 0 & 1 & 1 & 1 & 1 & 1 & 1 & 0 & 1 & 0 \\
\hline 33 & 1 & 1 & 0 & 1 & 1 & 0 & 0 & 1 & 0 & & 1 & 1 & 1 & 0 & 1 & 1 & 0 & 0 & 1 & 0 & 1 \\
\hline 34 & 1 & 1 & 1 & 1 & 0 & 1 & 0 & 1 & 1 & & 0 & 1 & 1 & 1 & 1 & 0 & 1 & 0 & 1 & 1 & 0 \\
\hline 35 & 1 & 0 & 0 & 0 & 0 & 0 & 1 & 0 & 0 & & 1 & 1 & 0 & 0 & 0 & 0 & 0 & 1 & 0 & 0 & 1 \\
\hline
\end{tabular}

Table 4--- Memorised Pattern Set for Feed Forward Network trained by Back Propagation

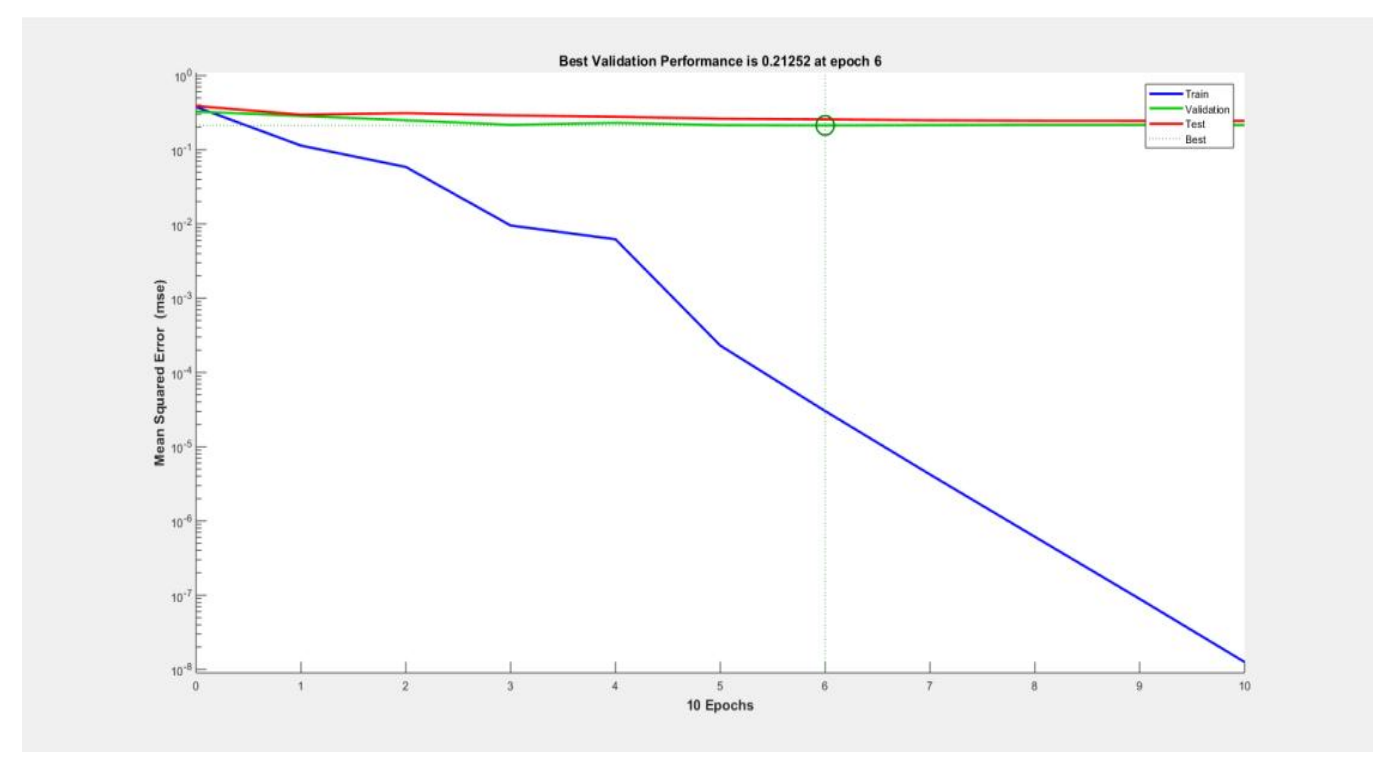

Figure2 . Shows The Performance Curve of Neural Network

\section{Validation by Test Pattern :}

The network was successfully trained and when we fed the test patterns, the result so obtained are shown in table below.

\section{Test Pattern}




\begin{tabular}{|l|l|l|l|l|l|l|l|l|l|}
\hline 1 & 0 & 0 & 0 & 0 & 0 & 0 & 1 & 0 & 1 \\
\hline 0 & 0 & 1 & 0 & 0 & 1 & 1 & 0 & 1 & 0 \\
\hline 0 & 0 & 0 & 0 & 0 & 0 & 1 & 0 & 0 & 1 \\
\hline 0 & 1 & 1 & 1 & 1 & 1 & 0 & 1 & 1 & 1 \\
\hline
\end{tabular}

Output for Test Pattern (bit by bit) By the Network.

\begin{tabular}{|c|c|c|c|c|c|}
\hline 0.86393 & 0.17279 & 0.81072 & -0.95569 & -0.92987 & -0.89381 \\
\hline 0.89422 & -0.74509 & -0.94759 & -0.82977 & -0.76931 & -0.17444 \\
\hline-0.39197 & 0.94074 & 0.83381 & 0.94886 & 0.92379 & 0.8375 \\
\hline-0.12771 & 0.24354 & 0.29411 & 0.92561 & -0.81538 & -0.30699 \\
\hline $\begin{array}{c}-0.26994 \\
\end{array}$ & -0.036851 & 0.16146 & 0.20129 & -0.32514 & -0.34186 \\
\hline-0.26994 & -0.036851 & 0.16146 & 0.20129 & -0.32514 & -0.34186 \\
\hline 0.34985 & -0.90363 & -0.21114 & -0.38456 & 0.39822 & -0.38826 \\
\hline-0.1756 & -0.17135 & -0.20477 & 0.13256 & 0.15689 & -0.058926 \\
\hline 0.052763 & 0.82731 & -0.065994 & -0.83008 & 0.15434 & 0.127 \\
\hline-0.30175 & 0.38736 & 0.052211 & 0.070218 & -0.0083134 & -0.40444 \\
\hline-0.32589 & -0.19489 & 0.0052044 & 0.026907 & -0.93278 & 0.35184 \\
\hline 0.90919 & -0.31459 & 0.2593 & -0.28413 & -0.91 & 0.33599 \\
\hline 0.21378 & -0.025403 & 0.26298 & -0.00509 & -0.39976 & -0.8233 \\
\hline 0.73362 & 0.27761 & -0.35748 & -0.032807 & 0.11863 & 0.038341 \\
\hline 0.075215 & -0.15065 & 0.37533 & 0.3742 & -0.32951 & 0.3084 \\
\hline-0.38729 & 0.9328 & 0.020015 & -0.090406 & 0.20527 & -0.1821 \\
\hline 0.37804 & -0.91504 & 0.18206 & 0.2553 & -0.32821 & 0.25141 \\
\hline 0.15471 & -0.21882 & -0.21357 & -0.069184 & 0.80712 & 0.90215 \\
\hline-0.21752 & 0.15662 & 0.17986 & 0.073244 & 0.34196 & -0.21752 \\
\hline-0.18171 & -0.15355 & -0.90093 & 0.83037 & 0.094684 & 0.14227 \\
\hline-0.094379 & -0.16688 & 0.022904 & 0.12374 & -0.37663 & 0.36544 \\
\hline 0.80574 & 0.29664 & -0.0085408 & 0.17461 & 0.32188 & 0.80092 \\
\hline 0.20103 & 0.02024 & -0.31115 & -0.2894 & 0.08821 & -0.32378 \\
\hline
\end{tabular}


International Journal of Innovative Technology and Exploring Engineering (IJITEE) ISSN: 2278-3075, Volume-8, Issue-9S3, July 2019

\begin{tabular}{|c|c|c|c|c|c|}
\hline-0.036538 & -0.27211 & 0.02103 & -0.92587 & -0.057359 & 0.077398 \\
\hline 0.34303 & 0.93417 & 0.2084 & -0.33183 & -0.39826 & 0.14368 \\
\hline-0.11019 & -0.80139 & -0.18409 & 0.2271 & 0.21161 & 0.17593 \\
\hline 0.2143 & -0.18331 & 0.28035 & 0.26513 & -0.31871 & -0.24507 \\
\hline-0.27264 & -0.36816 & -0.13653 & -0.34782 & -0.056755 & 0.026235 \\
\hline-0.25473 & 0.22406 & 0.054299 & 0.079148 & -0.97791 & -0.18663 \\
\hline-0.15125 & -0.31519 & -0.0131 & 0.94957 & 0.36588 & -0.14673 \\
\hline-0.045166 & -0.085953 & -0.068903 & -0.20416 & -0.30334 & -0.32353 \\
\hline-0.2958 & 0.30919 & 0.93587 & 0.81712 & 0.074728 & -0.037872 \\
\hline-0.33472 & -0.23202 & 0.12405 & -0.1536 & 0.15476 & 0.22397 \\
\hline 0.38411 & 0.039047 & -0.11226 & 0.5011 & -0.041316 & -0.11629 \\
\hline 0.045994 & -0.17944 & -0.35453 & -0.03003 & -0.09832 & 0.39248 \\
\hline 0.12324 & -0.32306 & 0.077038 & -0.1627 & 0.31153 & -0.29617 \\
\hline 0.47252 & -0.18373 & 0.026629 & 0.27935 & -0.3734 & -0.38674 \\
\hline-0.056507 & -0.32645 & 0.47965 & -0.015406 & 0.13766 & 0.14539 \\
\hline 0.39748 & -0.94506 & 0.23669 & -0.12832 & 0.25321 & 0.12927 \\
\hline 0.9192 & 0.87369 & 0.91252 & -0.32134 & -0.94723 & -0.22002 \\
\hline-0.31483 & -0.31017 & -0.039011 & -0.026245 & 0.098319 & -0.35762 \\
\hline 0.25676 & -0.24953 & -0.24072 & 0.1821 & 0.046442 & 0.20381 \\
\hline 0.17207 & 0.33167 & -0.95766 & 0.31598 & -0.076024 & -0.43502 \\
\hline 0.16071 & 0.0049736 & -0.3621 & 0.16954 & -0.061718 & -0.34223 \\
\hline-0.089626 & -0.23779 & -0.29253 & 0.90588 & -0.80646 & -0.21171 \\
\hline-0.1422 & 0.26198 & -0.33945 & -0.3657 & -0.056139 & -0.035928 \\
\hline-0.26383 & -0.30569 & -0.14013 & 0.31039 & -0.28046 & 0.32772 \\
\hline-0.90531 & -0.043533 & -0.38101 & -0.1229 & 0.92769 & 0.31905 \\
\hline-0.25371 & -0.2567 & 0.29877 & -0.027529 & 0.22824 & -0.32488 \\
\hline 0.085509 & 0.13576 & -0.31928 & 0.10535 & 0.2304 & 0.055341 \\
\hline-0.064298 & 0.26273 & 0.40254 & -0.17709 & 0.93413 & -0.064298 \\
\hline
\end{tabular}


Recognition of Text CAPTCHA by using Back Propagation Algorithm of Artificial Neural network

\begin{tabular}{|c|c|c|c|c|c|}
\hline $\begin{array}{l}-0.8401 \\
\end{array}$ & 0.14963 & 0.82339 & 0.13571 & -0.8401 & 0.14963 \\
\hline 0.072292 & 0.016923 & -0.30386 & -0.038694 & 0.049022 & 0.91992 \\
\hline-0.089454 & 0.22156 & 0.013522 & 0.10553 & -0.089454 & 0.12287 \\
\hline-0.94948 & 0.91227 & -0.10376 & 0.21526 & 0.25781 & -0.94948 \\
\hline 0.35975 & -0.096596 & 0.24154 & 0.37091 & 0.041005 & 0.38636 \\
\hline 0.21382 & 0.01629 & 0.36824 & -0.10839 & -0.35548 & 0.38864 \\
\hline 0.19427 & 0.14187 & -0.045229 & -0.092243 & 0.33666 & 0.92923 \\
\hline-0.31361 & -0.10395 & 0.21082 & 0.37117 & 0.94455 & -0.31361 \\
\hline 0.25819 & 0.30561 & -0.18357 & -0.30937 & 0.25819 & 0.30561 \\
\hline 0.11379 & -0.36613 & 0.31021 & -0.28586 & -0.30391 & 0.32259 \\
\hline-0.25118 & -0.012844 & -0.067866 & 0.020552 & $2.1816 \mathrm{e}$ & -0050.459 \\
\hline-0.93498 & -0.30055 & 0.095804 & 0.13391 & -0.025782 & 0.26283 \\
\hline 0.23696 & 0.14946 & -0.015458 & 0.31202 & 0.23696 & 0.21156 \\
\hline 0.27109 & 0.030159 & -0.24966 & -0.10156 & -0.067796 & -0.33314 \\
\hline-0.2837 & -0.021222 & 0.15748 & 0.25141 & -0.075835 & -0.030733 \\
\hline 0.98377 & -0.36707 & -0.15832 & 0.27852 & 0.21567 & -0.36492 \\
\hline-0.021643 & 0.12125 & -0.37836 & -0.057049 & 0.24973 & 0.29304 \\
\hline 0.316 & -0.32205 & $\begin{array}{l}-0.93689 \\
\end{array}$ & -0.24714 & -0.32106 & 0.27282 \\
\hline 0.95726 & 0.28701 & -0.29929 & 0.91029 & -0.31957 & -0.33879 \\
\hline-0.38571 & -0.050864 & 0.38698 & -0.010612 & 0.38683 & 0.28706 \\
\hline-0.22068 & -0.017183 & 0.18729 & -0.24783 & -0.15185 & 0.15485 \\
\hline 0.12224 & -0.055144 & -0.28687 & 0.28803 & -0.13151 & -0.3188 \\
\hline-0.42606 & 0.059127 & 0.38723 & 0.22398 & 0.25991 & 0.28467 \\
\hline-0.17445 & 0.35471 & 0.17354 & $-0.32161 ;$ & -0.36658 & -0.010478 \\
\hline-0.15356 & 0.10439 & -0.37253 & -0.37672 & -0.19823 & 0.99109 \\
\hline 0.12699 & 0.11246 & -0.047294 & -0.078988 & -0.38835 & 0.20461 \\
\hline 0.18028 & 0.10993 & -0.085823 & 0.27841 & -0.99178 & -0.095244 \\
\hline-0.27859 & -0.46111 & 0.12382 & -0.33052 & -0.32327 & 0.03457 \\
\hline-0.45927 & -0.30128 & -0.012689 & $\begin{array}{l}-0.18791 \\
\end{array}$ & -0.15275 & -0.037608 \\
\hline \multicolumn{6}{|c|}{1455} \\
\hline
\end{tabular}


International Journal of Innovative Technology and Exploring Engineering (IJITEE) ISSN: 2278-3075, Volume-8, Issue-9S3, July 2019

\begin{tabular}{|c|c|c|c|c|c|}
\hline 0.83105 & -0.089213 & -0.062046 & -0.34596 & -0.27027 & 0.92109 \\
\hline 0.50405 & 0.0097021 & -0.069704 & 0.20426 & 0.13417 & -0.077091 \\
\hline 0.054585 & 0.10206 & 0.15796 & -0.24052 & -0.35149 & 0.38989 \\
\hline 0.99357 & -0.19287 & 0.28359 & -0.002173 & -0.011893 & 0.084048 \\
\hline 0.18564 & 0.11085 & -0.44567 & -0.98702 & -0.1274 & 0.30668 \\
\hline 0.37267 & 0.13116 & -0.22313 & 0.13812 & 0.071448 & 0.40888 \\
\hline 0.0096557 & -0.34859 & -0.093661 & 0.32221 & 0.29161 & 0.39687 \\
\hline-0.24545 & -0.91808 & 0.15401 & 0.21373 & 0.29843 & 0.42377 \\
\hline-0.067849 & 0.29185 & 0.35933 & -0.27564 & -0.90152 & -0.15165 \\
\hline-0.17934 & 0.29206 & -0.36885 & 0.046415 & -0.09322 & -0.22317 \\
\hline-0.18453 & -0.35989 & 0.069377 & 0.29509 & 0.21143 & 0.11719 \\
\hline 0.19108 & 0.050353 & 0.075952 & 0.32077 & -0.24433 & -0.13387 \\
\hline 0.30851 & -0.92065 & -0.083069 & -0.11652 & -0.83545 & 0.19707 \\
\hline-0.25969 & -0.24597 & -0.31101 & -0.232 & 0.98746 & 0.29283 \\
\hline-0.38064 & 0.93546 & 0.90242 & -0.36949 & -0.1635 & 0.3013 \\
\hline-0.27372 & -0.2293 & 0.3968 & -0.50052 & 0.030932 & 0.32677 \\
\hline 0.39201 & 0.21728 & -0.022788 & 0.21171 & -0.3029 & 0.2836 \\
\hline 0.25153 & -0.077874 & 0.41631 & 0.065454 & -0.31295 & 0.41211 \\
\hline 0.070798 & -0.15391 & -0.36856 & 0.35612 & 0.096797 & -0.30809 \\
\hline-0.25845 & -0.15482 & 0.29448 & -0.25125 & 0.19508 & -0.32576 \\
\hline-0.24519 & -0.17577 & 0.37795 & 0.12109 & -0.3948 & 0.17338 \\
\hline 0.15617 & -0.287 & -0.27726 & -0.20679 & 0.16853 & -0.24237 \\
\hline-0.34312 & -0.35344 & -0.21485 & -0.26858 & 0.033399 & -0.26535 \\
\hline-0.24991 & 0.089004 & 0.21239 & 0.34814 & 0.036862 & 0.25824 \\
\hline-0.38252 & 0.25738 & 0.52853 & 0.92738 & 0.26654 & 0.1988 \\
\hline-0.016794 & 0.18753 & 0.060316 & 0.1055 & 0.38203 & -0.36406 \\
\hline-0.441 & 0.10269 & 0.17069 & 0.31423 & -0.3953 & 0.11593 \\
\hline-0.1153 & -0.22586 & 0.36585 & -0.24702 & -0.10359 & 0.19101 \\
\hline
\end{tabular}


Recognition of Text CAPTCHA by using Back Propagation Algorithm of Artificial Neural network

\begin{tabular}{|c|c|c|c|c|c|}
\hline-0.19905 & 0.1467 & -0.05352 & -0.26212 & 0.25218 & 0.038696 \\
\hline-0.066563 & -0.38948 & 0.081674 & -0.29533 & -0.9147 & 0.037977 \\
\hline-0.80705 & -0.9651 & -0.34946 & -0.33069 & -0.066902 & -0.29974 \\
\hline-0.15643 & 0.15766 & -0.25772 & 0.32268 & 0.14335 & 0.078804 \\
\hline-0.25049 & -0.13776 & 0.29686 & 0.28797 & 0.095051 & 0.38197 \\
\hline 0.35723 & 0.30627 & 0.15374 & 0.10822 & -0.085382 & 0.15512 \\
\hline-0.28775 & 0.34191 & 0.02348 & 0.35839 & -0.35888 & -0.23408 \\
\hline-0.4227 & 0.33313 & 0.19124 & -0.238 & -0.34089 & -0.91734 \\
\hline 0.26057 & -0.044604 & -0.26615 & -0.18556 & 0.36451 & -0.90279 \\
\hline 0.39092 & $\begin{array}{l}-0.3039 \\
\end{array}$ & 0.044788 & 0.37231 & 0.011934 & -0.9365 \\
\hline 0.029567 & 0.19025 & 0.26678 & -0.82454 & 0.27325 & 0.035243 \\
\hline-0.21142 & 0.28822 & 0.31021 & -0.19141 & -0.21187 & 0.027332 \\
\hline-0.098016 & -0.32761 & 0.1499 & 0.21178 & 0.12178 & 0.39129 \\
\hline-0.90195 & 0.15125 & -0.35097 & -0.35975 & 0.098953 & -0.3807 \\
\hline-0.042702 & 0.014915 & 0.24079 & 0.16175 & -0.34479 & 0.37754 \\
\hline-0.31458 & -0.08886 & -0.91355 & -0.13509 & -0.25181 & -0.36315 \\
\hline-0.0091389 & -0.39776 & 0.17928 & 0.29355 & 0.19761 & -0.14076 \\
\hline-0.37003 & -0.16464 & 0.32682 & 0.24354 & 0.16167 & -0.37693 \\
\hline-0.1974 & 0.93836 & -0.19199 & -0.052739 & 0.093635 & -0.34615 \\
\hline-0.18636 & -0.39872 & 0.37447 & -0.070003 & 0.18344 & 0.15493 \\
\hline 0.25363 & -0.0016099 & -0.21075 & 0.32063 & -0.91728 & -0.32574 \\
\hline 0.93201 & -0.37649 & -0.23433 & -0.32678 & -0.099137 & 0.16387 \\
\hline 0.97497 & 0.86118 & 0.27605 & 0.074995 & -0.37472 & -0.014905 \\
\hline-0.10156 & -0.0047461 & 0.12395 & 0.037982 & 0.35322 & -0.084116 \\
\hline $\begin{array}{l}-0.97896 \\
\end{array}$ & 0.025687 & 0.13848 & -0.25844 & -0.18125 & 0.20342 \\
\hline 0.25555 & 0.98322 & 0.27033 & 0.0403 & -0.13816 & -0.27336 \\
\hline 0.39514 & -0.21371 & -0.17305 & 0.50312 & 0.028248 & -0.3383 \\
\hline 0.24201 & 0.032934 & 0.18429 & 0.19273 & 0.96229 & -0.11198 \\
\hline 0.32427 & -0.32613 & 0.16916 & 0.20513 & 0.30014 & -0.036955 \\
\hline \multicolumn{6}{|c|}{1457} \\
\hline
\end{tabular}




\begin{tabular}{|c|c|c|c|c|c|}
\hline 0.91747 & 0.24144 & -0.17993 & 0.032449 & 0.28665 & 0.91747 \\
\hline 0.06074 & 0.061297 & 0.97661 & 0.14932 & -0.12446 & -0.50052 \\
\hline-0.18433 & 0.29301 & 0.57295 & -0.435 & 0.26532 & 0.2676 \\
\hline 0.20928 & 0.20756 & -0.096083 & -0.92958 & -0.1803 & 0.24563 \\
\hline-0.56966 & 0.053807 & 0.16809 & 0.19797 & 0.29457 & -0.0057674 \\
\hline-0.0010209 & 0.015449 & 0.30483 & -0.35031 & 0.23934 & 0.17763 \\
\hline-0.47175 & 0.22685 & -0.21546 & 0.2801 & -0.076297 & 0.047214 \\
\hline 0.16554 & 0.090785 & -0.12729 & -0.44912 & -0.33654 & 0.15731 \\
\hline 0.1304 & 0.25687 & -0.08074 & -0.38696 & 0.00050214 & 0.16701 \\
\hline 0.089509 & -0.41989 & 0.10878 & -0.079077 & -0.021866 & -0.42677 \\
\hline-0.13054 & -0.37519 & 0.41604 & 0.038118 & -0.34326 & 0.33879 \\
\hline-0.83294 & 0.29958 & 0.26525 & -0.10081 & -0.39891 & -0.35539 \\
\hline-0.080561 & 0.19136 & -0.11631 & -0.35512 & -0.29829 & 0.073249 \\
\hline-0.061909 & -0.28086 & -0.053003 & 0.09401 & 0.0084871 & 0.23893 \\
\hline-0.031225 & 0.13821 & -0.3552 & -0.42362 & 0.11984 & -0.12229 \\
\hline 0.1560. & -0.39944 & 15047 & 0.1323 & 0.021657 & -0.30671 \\
\hline-0.059203 & -0.10953 & -0.43177 & -0.20805 & -0.22899 & 0.089842 \\
\hline
\end{tabular}

Table4 shows The Validation of test Patterns without any training

\section{DISCUSSION}

With the above experiment has been shown that binary bits of text CAPTCHA can be stored in the network, it also indicates that the system is working properly for password validation and finding storing them using the model of Back Propagation of Artificial Neural Networks . Password strength is depending on the number of neurons in the model. According to performance curve the machine recognize the character of 10 epochs. Best validation performance is 21252 . Table 4 shows the memorized input patterns of the network. Table 2 shows that the system is able to generate data based on pattern mapped earlier during the training and give excellent results for patterns never trained. TRAINLM function is used to train the network which gives best result as compare to all other function. It takes minimum time to train the network and minimum number of iteration to train the network.
Performance curve shows that machine recognize the character in first 10 epochs.

\section{RESULTS}

This paper proposed a technique of CAPTCHA storing and recalling based on Back Propagation model of Artificial Neural Networks.. According to result of training the performance curve CAPTCHA is recognised very easily without any pre-processing and segmentation. Back Propagation model is introduced to improve network performance, which can recognize the different character of the CAPTCHA image with minimum number of iterations.

\section{REFERENCES}

1. Tomoka Azakami, Chihiro Shibata, Ryuya Uda, "Challenge of Deep Learning against CAPTCHA with Amodal Completion and Aftereffects by Colors" 2016 , 2157-0426. \& Sciences Publication 


\section{Recognition of Text CAPTCHA by using Back Propagation Algorithm of Artificial Neural network}

2. Shailaja Tingre, Debajyoti Mukhopadhyay, "An Approach for Segmentation of Characters in CAPTCHA", ICCCA 2015,978-14799-8890.

3. $\mathrm{Yu} \mathrm{Hu}{ }^{1,2}, \mathrm{Li} \mathrm{Chen}^{1,2^{*}}$, Jun Cheng ${ }^{3}$, “ A CAPTCHA recognition technology based on deep learning" IEEE 2018,978-1-5386.

4. Xiao Ling-Zi, ZHANG Yi-Chun , " A Case Study of Text -Based CAPTCHA Attacks", IEEE 2012, 978-0-7695.

5. Mengyuan Wang ${ }^{1}$, Yuliang Yang ${ }^{1}$, Mengyu Zhu ${ }^{2}$, Jiaming Liu $^{3}$, "CAPTCHA Identification Based on Convolution Neural Network”, IMCES 2018, 978-1-5386-1803.

6. Brian Cheung, "Convolutional Neural Networks Applied to Human Face Classification" International Conference on Machine Learning and Application, 2012, 978-0-7695 .

7. Yanping $\mathrm{Lv}^{1,2}$, Feipeng $\mathrm{Ca}^{1,2}$, Dazhen $\mathrm{Lin}^{1,2^{*}}$, Donglin Cao ${ }^{1,2^{*}}$, "Chinese Character CAPTCHA Recognition Based on Convolution Neural Network",IEEE 2016, 978-1-5090-0623.
8. Ye Wang, Yuanjiang Huang, Wu Zheng, Zhi Zhou, Debin Liu, Mi $\mathrm{Lu}$ "Combining convolutional neural network and self-adaptive algorithm to defeat synthetic multi-digit text-based CAPTCHA" IEEE 2017, 978-1-5090-5320.

9. Mengyun Tang, Haichang Gao, Yang Zhang, Yi Liu, Ping Zhang, Ping Wang “ Research on Deep Learning Techniques in Breaking Text -Based CAPTCHAs and Designing Image -Based CAPTCHA", IEEE Transaction on Information Forensics and Security, October-2018 Vol. 13 , No.-10.

10. Rafaqat Hussain, Hui Gao, Raiz Ahmed Shaikh, Shazia Parveen Soomro, " Recognition Based Segmentation of Connected Characters in Text Based CAPTCHAs" IEEE International Conference on Communication Software and Networks, 2016, 978-1-5090-1781.

11. Tao Zhang ${ }^{1}$, Honglei Zheng ${ }^{2}$, Lele Zhang ${ }^{3}$, “ Verification CAPTCHA Based on Deep Learning", Proceeding of the $37^{\text {th }}$ Chinese Control Conference “,July 25-27, 2018. 